\title{
On-Line Covering the Unit Cube by Cubes*
}

\author{
J. Januszewski and M. Lassak \\ Instytut Matematyki i Fizyki ATR, ul. Kaliskiego 7, \\ 85-791 Bydgoszcz, Poland \\ januszew@atr.bydgoszcz.pl \\ lassak@atr.bydgoszcz.pl
}

\begin{abstract}
We consider two on-line methods of covering the unit cube of Euclidean $d$-space by sequences of cubes. The on-line restriction means that we are given the next cube from the sequence only after the preceding cube has been put in place without the possibility of changing the placement. The first method enables on-line covering of the unit cube by an arbitrary sequence of cubes whose total volume is at least $3 \cdot 2^{d}-4$. The second method is more complicated, but, asymptotically, as $d$ tends to infinity, it yields an efficiency of the order of magnitude $2^{d}$ with factor 1. So, asymptotically, it is as good as the best possible non-on-line method of covering the unit cube by cubes.
\end{abstract}

\section{Introduction}

Denote by $E^{d}$ the Euclidean $d$-space. We say that a sequence $C_{1}, C_{2}, \ldots$ of sets of $E^{d}$ permits a covering of a set $C \subset E^{d}$ if there are rigid motions $\sigma_{i}$ such that $C$ is contained in the union of sets $\sigma_{i} C_{i}$, where $i=1,2, \ldots$ In [3] Groemer presents a survey of results on coverings of convex bodies by sequences of convex bodies.

The present paper considers covering methods which work under the restriction that we learn $C_{i}$, where $i>1$, only after $C_{i-1}$ has been placed, i.e., only after the motion $\sigma_{i-1}$ has been irreversibly provided. At the beginning we are given $C_{1}$. Such a covering is called an on-line covering by analogy to the on-line packing discussed in [6].

Kuperberg [5] proved that the unit cube $I^{d}$ can be on-line covered by an arbitrary sequence of cubes of total volume at least $4^{d}$. Our aim is to improve this

\footnotetext{
* This research was supported in part by Komitet Badań Naukowych (Committee of Scientific Research), Grant Number 220059203.
} 
estimate. We present two on-line methods of covering $I^{d}$ by cubes. The first method makes possible an on-line covering of $I^{d}$ by every sequence of cubes of total volume at least $3 \cdot 2^{d}-4$. The second method is more sophisticated but it enables an on-line covering of $I^{d}$ by every sequence of cubes whose total volume is a number of the order of magnitude of $2^{d}$ with factor 1 , the same as for the bound $2^{d}-1$ of the best non-on-line method of Groemer [2] and Bezdek and Bezdek [1].

\section{The Method of the Current Bottom}

Points of the unit cube

$$
I^{d}=\left\{\left(x_{1}, \ldots, x_{d}\right): 0 \leq x_{1} \leq 1, \ldots, 0 \leq x_{d} \leq 1\right\}
$$

will be successively on-line covered by a sequence $Q_{1}, Q_{2}, \ldots$ of $d$-dimensional cubes. In every $Q_{i}$, when we learn it, we find the greatest cube $K_{i}$ whose side is of length of the form $2^{-r}$, where $r$ is a nonnegative integer. Then we find the greatest number $b_{i} \leq 1$ such that every point of $I^{d}$ whose last coordinate is smaller than $b_{i}$ has been covered by the preceding cubes, i.e., by cubes $K_{j}$ such that $j<i$. The set of all points of the cube $I^{d}$ having the last coordinate $b_{i}$ is called the ith bottom or the current bottom of the cube. Of course, if $b_{i}>0$, then the $i$ th bottom is covered by cubes $K_{j}$, where $j<i$. A point of the $i$ th bottom is called a surface point if no point with the last coordinate greater and with the other coordinates unchanged has been covered by cubes $K_{j}$, where $j<i$. We find a rigid motion $\sigma_{i}$ such that $\sigma_{i} K_{i}$ contains a surface point of the $i$ th bottom and that $\sigma_{i} K_{i}$ has the form

$\left\{\left(x_{i}, \ldots, x_{d}\right): a_{k} 2^{-r} \leq x_{k} \leq\left(a_{k}+1\right) 2^{-r}\right.$ for $k=1, \ldots, d-1$ and $\left.b_{i} \leq x_{d} \leq b_{i}+2^{-r}\right\}$,

where $2^{-r}$ is the side of $K_{i}$ and where $a_{1}, \ldots, a_{d-1} \in\left\{0, \ldots, 2^{r}-1\right\}$. Observe that the prescription guarantees that at every stage there are surface points of the current bottom. If $b_{n}=1$ for an index $n$, then we can stop the covering process; at this moment $I^{d}$ has been covered by cubes $\sigma_{1} Q_{1}, \ldots, \sigma_{n-1} Q_{n-1}$. The method just described is called the method of the current bottom.

Theorem 1. The method of the current bottom enables on-line covering of the unit cube by an arbitrary sequence of cubes of total volume at least $3 \cdot 2^{d}-4$.

Proof. Obviously, every sequence containing at least one cube with sides of length at least 1 permits a covering of $I^{d}$ by the method of the current bottom. So we can assume that all cubes $Q_{1}, Q_{2}, \ldots$ have sides smaller than 1 .

For every $i \in\{1,2, \ldots\}$ the ratio of the volume $\left|Q_{i}\right|$ to the volume $\left|K_{i}\right|$ is smaller than $2^{d}$. Consequently, it is sufficient to show that the unit cube can be on-line covered by every sequence of cubes $K_{1}, K_{2}, \ldots$ of total volume at least $3-4 \cdot 2^{-d}$ whose sides are of the form $2^{-r}$, where $r$ is a positive integer.

Let $G_{i}(r)$ be a set containing a surface point of the $i$ th bottom and having the 
form

$$
\left\{\left(x_{1}, \ldots, x_{d}\right): a_{k} 2^{-r} \leq x_{k} \leq\left(a_{k}+1\right) 2^{-r} \text { for } k=1, \ldots, d-1 \text { and } b_{i} \leq x_{d}\right\}
$$

where $r$ is a nonnegative integer and $a_{1}, \ldots, a_{d-1} \in\left\{0, \ldots, 2^{r}-1\right\}$. Denote by $H_{i}(r)$ the part of $G_{i}(r)$ which is covered by cubes $\sigma_{j} K_{j}$, where $j<i$. The volume of $H_{i}(r)$ is smaller than the sum of the volumes of $2^{d-1}-1$ cubes of each of the sides $2^{-r-1}, 2^{-r-2}, \ldots$ Applying the formula for the sum of the geometric sequence we obtain that

$$
\left|H_{i}(r)\right|<\frac{2^{d-1}-1}{2^{d}-1} 2^{-r d}
$$

Putting $r=0$ in (1) we get

$$
\left|H_{i}(0)\right|<\frac{2^{d-1}-1}{2^{d}-1} \quad \text { for every } \quad i \in\{1,2, \ldots\} .
$$

From (1) we conclude that the volume of the common part of the cube $\sigma_{i} K_{i}$ and of the union of cubes $\sigma_{j} K_{j}$, where $j<i$, is smaller than

$$
\frac{2^{d-1}-1}{2^{d}-1}\left|K_{i}\right|
$$

Consequently, the region covered by $\sigma_{i} K_{i}$ for the first time (i.e., covered by $\sigma_{i} K_{i}$ but not covered by the union of $\sigma_{j} K_{j}$, where $j<i$ ) is of volume greater than $1-\left(2^{d-1}-1\right) /\left(2^{d}-1\right)=2^{d-1} /\left(2^{d}-1\right)$ times the volume of $K_{i}$. If we suppose that $b_{i}<1$ for every $i \in\{1,2, \ldots\}$, then the sum of the volumes of the regions covered by the cubes $\sigma_{i} K_{i}$ for the first time, where $i=1,2, \ldots$, is over

$$
\left(3-4 \cdot 2^{-d}\right) \frac{2^{d-1}}{2^{d}-1}=1+\frac{2^{d-1}}{2^{d}-1}
$$

This and the assumption that all $b_{i}$ are smaller than 1 lead to a contradiction with (2). Thus we conclude the existence of a positive integer $n$ such that $b_{n}=1$. So a finite number of cubes of our sequence covers the whole $I^{d}$.

\section{The Method of the Current Bottom and Top}

The idea of the method of the current bottom and top is in selecting the cubes $K_{i}$ in the cubes $Q_{i}, i=1,2, \ldots$, from amongst a larger family of cubes than in the method of the current bottom. So besides cubes of sides of lengths $1, \frac{1}{2}, \frac{1}{4}, \ldots$, as in the method of the current bottom, this time we are also looking for cubes of sides of lengths $\frac{1}{4} \sqrt{2}, \frac{1}{8} \sqrt{2}, \ldots$. The first kind of cubes are used for placing them 
starting from the bottom of $I^{d}$, and the second kind starting from the top of $I^{d}$. The advantage is that, for cubes $Q_{i}$ of sides less than $\frac{1}{2} \sqrt{2}$, the crucial factor $2^{d}$ from the method of the current bottom is now lessened to the factor $(\sqrt{2})^{d}$. However, we cannot lessen the factor for cubes of side between $\frac{1}{2} \sqrt{2}$ and 1 . For such cubes we apply another trick. Here are the details.

We on-line cover points of $I^{d}$ by a sequence $Q_{1}, Q_{2}, \ldots$ of cubes. A cube of our sequence is called very big if its side is of length at least 1 , and it is called big if its side is of length at least $\frac{1}{2} \sqrt{2}$ but smaller than 1 .

In every $Q_{i}$, when we learn it, we find the greatest cube $N_{i}$ whose side is of a length of the form $2^{-r}$ or $2^{-r-3 / 2}$, where $r$ is a nonnegative integer. If the length of the side of $N_{i}$ is of the form $2^{-r}$, where $r$ is a nonnegative integer, then we put $B_{i}=N_{i}$ and $T_{i}=\varnothing$. If the side of $N_{i}$ has the form $2^{-r-3 / 2}$, where $r$ is a nonnegative integer, we put $B_{i}=\varnothing$ and $T_{i}=N_{i}$.

The sequence $B_{1}, B_{2}, \ldots$ is used for a covering of points of $I^{d}$ by the method of the current bottom described in the preceding section. The difference is that if $B_{i}=\varnothing$, we do not find the rigid motion $\sigma_{i}$ and we do not use $B_{i}$ for the covering.

The sequence $T_{1}, T_{2}, \ldots$ is used for covering $I^{d}$ by a method similar to the method of the current bottom which is called the method of the current top. Here is the description. For every $T_{i}$ we find the greatest nonnegative number $t_{i}$ such that every point of $I^{d}$ with the last coordinate greater than $1-t_{i}$ has been covered by cubes $T_{j}$, where $j<i$. The set of all points of $I^{d}$ having the last coordinate $1-t_{i}$ is called the ith top or the current top. A point of the ith top is called a surface point if no point with the last coordinate smaller and with the other coordinates unchanged has been covered by cubes $T_{j}$, where $j<i$. If $T_{i} \neq \varnothing$, we find a rigid motion $\sigma_{i}$ such that $\sigma_{i} T_{i}$ contains a surface point of the $i$ th top and that it has the form

$$
\begin{aligned}
& \left\{\left(x_{1}, \ldots, x_{d}\right): a_{k} 2^{-r-3 / 2} \leq x_{k} \leq\left(a_{k}+1\right) 2^{-r-3 / 2}\right. \\
& \left.\quad \text { for } k=1, \ldots, d-1 \text { and } 1-t_{i}-2^{-r-3 / 2} \leq x_{d} \leq 1-t_{i}\right\}
\end{aligned}
$$

where $2^{-r-3 / 2}$ is the side of $T_{i}$ and where $a_{1}, \ldots, a_{d-1} \in\left\{0,1, \ldots, 3 \cdot 2^{r}-1\right\}$. Observe that the method of the current top guarantees that there are always surface points of the current top.

The above prescription finds a rigid motion $\sigma_{i}$ for every index $i$ which moves one of the sets, $B_{i}$ or $T_{i}$. Every time we move the corresponding cube $Q_{i}$. When $b_{n}+t_{n} \geq 1$, we can stop the covering process; at this moment $I^{d}$ has been covered by cubes $\sigma_{1} Q_{1}, \ldots, \sigma_{n-1} Q_{n-1}$.

Lemma 1. The set $\left\{\left(x_{1}, \ldots, x_{d}\right): 0 \leq x_{1} \leq 1, \ldots, 0 \leq x_{d} \leq a\right\}$ can be covered by the method of the current bottom by every sequence of cubes whose sides are of the form $2^{-r}$, where $r \in\{1,2, \ldots\}$, if the total volume of the cubes is at least

$$
b(a)=\frac{2^{d}-1}{2^{d-1}}\left[a+\frac{2^{d-1}-1}{2^{d}-1}\right]
$$


Lemma 2. The set $\left\{\left(x_{1}, \ldots, x_{d}\right): 0 \leq x_{1} \leq 1, \ldots, a \leq x_{d} \leq 1\right\}$ can be covered by the method of the current top by every sequence of cubes whose sides are of the form $2^{-r-3 / 2}$, where $r \in\{1,2, .$.$\} , if the total volume of the cubes is at least$

$$
t(a)=\frac{2^{d}-1}{2^{d-1}}\left[(1-a)\left(\frac{3 \sqrt{2}}{4}\right)^{d-1}+\left(3^{d-1}-\frac{2^{d-1}}{2^{d}-1}\right)\left(\frac{\sqrt{2}}{4}\right)^{d}\right]
$$

We omit the proofs of Lemmas 1 and 2 because they are similar to the proof of Theorem 1.

Theorem 2. Every sequence of d-dimensional cubes of total volume at least $2^{d}+\left(\frac{4}{3} \sqrt{2}+\frac{2}{3}\right)\left(\frac{3}{2}\right)^{d}$ permits an on-line covering of $I^{d}$ by the method of the current bottom and top.

Proof. Of course, every sequence of cubes containing a very big cube or at least $2^{d}$ big cubes covers $I^{d}$ by the method of the current bottom and top. Further, we consider only sequences of cubes without very big cubes and with at most $2^{d}-1$ big cubes.

The linearity of $b(x)+t(x)$, where $b(x)$ and $t(x)$ are defined in Lemmas 1 and 2 , easily implies that the function $b(x)+t(x)$ is decreasing. Thus $b(a)+t(a) \leq$ $b(0)+t(0)$ for every $a$ between 0 and 1 . Consequently, we can cover $I^{d}$ by the sequence $Q_{1}, Q_{2}, \ldots$ by the method of the current bottom and top if the volume of the sequence $N_{1}, N_{2}, \ldots$ (defined in the description of the method) is at least $b(0)+t(0)$.

There are up to $2^{d}-1$ big cubes in the sequence $Q_{1}, Q_{2}, \ldots$ For every big cube $Q_{i}$ we have $\left|Q_{i}\right|-(\sqrt{2})^{d}\left|N_{i}\right|<1-\left(\frac{1}{2} \sqrt{2}\right)^{d}$. So $I^{d}$ can be covered by the method of the current bottom and top by every sequence of cubes $Q_{1}, Q_{2}, \ldots$ of total volume at least

$$
(\sqrt{2})^{d}[b(0)+t(0)]+\left(2^{d}-1\right)\left[1-\left(\frac{1}{2} \sqrt{2}\right)^{d}\right]<2^{d}+\left(4 \sqrt{2}+\frac{2}{3}\right)\left(\frac{3}{2}\right)^{d}
$$

More specific estimates resulting from the method of the current bottom and top are presented in [4].

\section{References}

1. A. Bezdek and K. Bezdek, Eine hinreichende Bedinung für die Uberdeckung des Einheitwürfels durch homothetische Exemplare im n-dimensionalen Raum, Beitr. Geom. Algebra 17 (1984), 5-21.

2. H. Groemer, Covering and packing properties of bounded sequences of convex sets, Mathematika 29 (1982), 18-31.

3. H. Groemer, Coverings and packings by sequences of convex sets, in Discrete Geometry and Convexity, Annals of the New York Academy of Science, vol. 440, 1985, pp. 262-278. 
4. J. Januszewski and M. Lassak, On-line covering the unit sequare by squares and the threedimensional unit cube by cubes, Demonstratio Math. 27 (1994) (to appear).

5. W. Kuperberg, On-line covering a cube by a sequence of cubes. Discrete Comput. Geom. 12 (1994), 83-90.

6. M. Lassak and J. Zhang, An on-line potato-sack theorem, Discrete Comput. Geom. 6 (1991), 1-7.

Received March 1, 1993, and in revised form September 29, 1993, and March 10, 1994. 